\title{
AN INEQUALITY FOR INCIDENCE MATRICES
}

\section{J. R. ISBELL}

This paper establishes an extremal property for finite projective planes; it is a characteristic property, if we disregard configurations containing fewer than four points. A finite plane consists of $k^{2}-k+1$ points, with lines each of which contains only $k$ points; nevertheless every two lines meet. This would not be remarkable if, say, we could define a "line" as any set of $k$ points containing a specified point $P_{0}$; but we must satisfy the homogeneity condition, that each point is contained in exactly $k$ lines. The theorem says that $k^{2}-k+1$ is the largest number of points which can be arranged in such a pattern.

A similar, almost characteristic, inequality has been established by de Bruijn and Erdös ${ }^{1}[1]$ : in a set of $n$ points one cannot find more than $n$ subsets, every two of which have exactly one common point. There are two trivial ways of constructing such a family for any $n$, but if there are to be four points, no three of which are on a line, then the finite projective planes are the only possibilities.

Both theorems generalize ${ }^{2}$ to the case in which a pair of lines must have more than one common point. The precise results are

Throrem 1. Suppose $v, k, \lambda$ are positive integers, and in a set of $v$ points certain distinct subsets are designated as lines, in such a way that

(a) Every two lines have at least $\lambda$ common points;

(b) The number of lines containing a given point is the same for all points; and

(c) No line contains more than $k$ points.

Then $\lambda(v-1) \leqq k^{2}-k$. In case of equality, the points and lines form a $(v, k, \lambda)$ configuration. If $\lambda=1$ and $k>2$, this is a finite projective plane.

Theorem 2. For $v>0$ and $\lambda \geqq 0$, a set of $v$ points contains no family of more than $v$ distinct nonempty subsets the intersection of any two of which consists of exactly $\lambda$ points.

In Theorem 2, the $(v, k, \lambda)$ configurations of course furnish examples. For $\lambda=0$ (we may extend the usual definition [2] to this trivial case) there are no exceptional cases of equality; for $\lambda=1$, the excep-

Received by the editors June 9, 1958 and, in revised form, August 7, 1958. [3].

${ }^{1}$ De Bruijn and Erdös do not draw the full conclusion in [1]; see Hall's review

2 I am indebted to J. P. Jans and H. J. Ryser for discussions which led to the conjecture and proof of Theorem 2 . 
tions are known; for $\lambda \geqq 2$, I do not know anything about exceptions. The proof of Theorem 1 will involve the

Lemma. Let $N$ be a finite set of objects, and $h, k, l$, and $\lambda$ positive integers. Suppose there are given a nonempty collection of subsets of $N$ called rows, and another nonempty collection of subsets called columns satisfying

(i) every row and column have at least $\lambda$ common elements;

(ii) no row has more than $h$ elements;

(iii) no column has more than k elements; and

(iv) each object lying in $r$ rows is in exactly $l-r$ columns. Then the number of elements of $N$ is at most $h k / \lambda$. If equality is attained then every row has exactly h elements, every column has exactly $k$ elements, every row and column have exactly $\lambda$ common elements, and every element is in the same number $r$ of rows.

Proof. This will reduce to the following special case of Cauchy's inequality, for $n$ real numbers $r_{i}: n \sum r_{i}^{2} \geqq\left(\sum r_{i}\right)^{2}$. The left side is the square of the product of the norms of the vectors $\left(r_{1}, \cdots, r_{n}\right)$ and $(1, \cdots, 1)$, the right side the square of their scalar product. Note that equality can occur only if all $r_{i}$ are equal. For the reduction, let $s$ be the number of rows, $t$ the number of columns. Number the objects $i=1,2, \cdots, n$. Let $r_{i}$ be the number of rows containing object $i$, so that by assumption (iv) object $i$ is in $l-r_{i}$ columns. Then assumption (i) implies $\sum r_{i}\left(l-r_{i}\right) \geqq \lambda s t$; (ii) implies $\sum r_{i} \leqq s h$, and (iii), $\sum\left(l-r_{i}\right) \leqq t k$. Writing $h k / \lambda$ as $(s h)(t k) / \lambda s t$, we see that it is at least as great as

$$
\frac{\left(\sum r_{i}\right)\left(n l-\sum r_{i}\right)}{l \sum r_{i}-\sum r_{i}^{2}} .
$$

Here equality is possible only if there is equality in each instance of (i), (ii), and (iii). It remains only to check $n l \sum r_{i}-n \sum r_{i}^{2} \leqq n l \sum r_{i}$ $-\left(\sum r_{i}\right)^{2}$, which follows from Cauchy's inequality as written above. Thus the proof is complete.

Proof OF Theorem 1 . In case $k \leqq \lambda$, the theorem asserts $v \leqq k$; we leave the proof as an exercise for the reader ${ }^{3}$ and assume $k>\lambda$. Select any one of the points, $p$. The lemma applies to the $v-1$ remaining points if we define a row to be a set which, with $p$ added, would make a line, and a column to be a line not containing $p$. Here $h=k-1$ and $l$ is the number of lines containing any given point. The lemma gives us the desired inequality $v-1 \leqq k(k-1) / \lambda$.

${ }^{3}$ This is the only case for which we need the condition that the lines are distinct. 
In case of equality the lemma tells us that every line in the original configuration contains exactly $k$ points and every pair of lines has exactly $\lambda$ common points. Moreover, the total number $w$ of lines can be determined as follows. Any one line has $k$ points on it; each of these points is on $l-1$ other lines, and in this count every other line has been counted $\lambda$ times. Thus $k(l-1)=\lambda(w-1)$, or $w=k(l-1) / \lambda+1$. Now counting all incidences of points with lines, we have $w k=v l$. Substituting the values of $w$ and $v$, we find $k(\lambda-k)=l(\lambda-k)$; since $k>\lambda$, this implies $k=l$ and $w=v$. It is known [2] that this implies that every two points are joined by exactly $\lambda$ lines. That is, we have a $(v, k, \lambda)$ configuration, and the proof is complete.

Proof of Theorem 2. The case $\lambda=0$ and the case that there is only one line are obvious. Let there be $w$ lines, $w \geqq 2, \lambda \geqq 1$. The proof will reduce to the proposition that the following $w$ by $w$ square matrix $\left(b_{i j}\right)$ is nonsingular. Count the lines $i=1, \cdots, w$, and the points $j=1, \cdots, v$, and let $b_{i i}$ be the number of points in the $i$ th line, with $b_{i j}=\lambda$ for $i \neq j$. Note that $b_{i i} \geqq \lambda$, and there is at most one index $i$ for which $b_{i i}=\lambda$; for a line having only $\lambda$ points must be a subset of every other line. If $\left(b_{i j}\right)$ were singular there would be a nonzero $w$-vector $\left(x_{i}\right)$ satisfying the $m$ equations with positive coefficients, $b_{j j} x_{j}+\sum_{i \neq j} \lambda x_{i}=0$. Subtracting the $k$ th equation from the $j$ th, we have $\left(b_{j j}-\lambda\right) x_{j}=\left(b_{k k}-\lambda\right) x_{k}$. If $b_{i i}>\lambda$ in all cases, then all $x_{i}$ have the same sign and hence all $x_{i}$ vanish. If $b_{i i}=\lambda$ in one case, then $\left(b_{j j}-\lambda\right) x_{j}$ $=0$ for all $j$ and hence $x_{j}=0$ for $j \neq i$. But then the $i$ th equation is $b_{i i} x_{i}=0$; since $b_{i i}>0, x_{i}=0$.

If we define the $w$ by $v$ matrix $\left(a_{i j}\right)$ by $a_{i j}=1$ when the $i$ th line contains the $j$ th point, $a_{i j}=0$ otherwise, the hypothesis of the theorem says that the matrix product $\left(a_{i j}\right)\left(a_{j i}\right)$ is exactly the matrix $\left(b_{i j}\right)$ above. Since the rank of $\left(a_{i j}\right)$ cannot exceed $v$, the nonsingularity of $\left(b_{i j}\right)$ implies $v \geqq w$.

\section{REFERENCES}

1. N. G. de Bruijn and P. Erdös, On a combinatorial problem, Indag. Math. vol. 10 (1949) pp. 421-423.

2. S. Chowla and H. J. Ryser, Combinatorial problems, Canad. J. Math. vol. 2 (1950) pp. 93-99.

3. M. Hall, Math. Reviews vol. 10 (1949) p. 424.

UNIVERSITY OF WASHINGTON 